\title{
Litchi Flowering is Regulated by Expression of Short Vegetative Phase Genes
}

\author{
Jiaqi Hu \\ Guangdong Litchi Engineering Research Center, College of Horticulture, South China Agricultural \\ University, Guangzhou 510642, China
}

\author{
Hye-Ji Kim \\ Department of Horticulture and Landscape Architecture, Purdue University, West Lafayette, IN \\ 47907-2010

\begin{abstract}
Houbin Chen and Biyan Zhou ${ }^{1}$
Guangdong Litchi Engineering Research Center, College of Horticulture, South China Agricultural University, Guangzhou 510642, China
\end{abstract}

\begin{abstract}
Additional INDEx words. apical meristem, brassinosteroid, floral repressor, LcSVP, Litchi chinensis, low temperature
ABSTRACT. Short vegetative phase (SVP), a MADS-domain transcription factor, was shown to act as a repressor of flowering in arabidopsis (Arabidopsis thaliana). Although the role of $S V P$ s in flowering is well characterized in the model plant arabidopsis, little is known in evergreen woody litchi (Litchi chinensis). In this study, three litchi $S V P$ homologs ( $L c S V P 1, L c S V P 2$, and $L c S V P 3)$ were cloned, and the bioinformatic analysis of the $L c S V P$ s was carried out to identify their molecular characteristics. Their expression patterns in the apical meristem (AM) during the transition from vegetative to reproductive phase were studied under natural flowering inductive conditions. Also, brassinosteroid (BR) treatment under low temperature conditions was performed to elucidate the role of $L c S V P$ s in the BR-regulated flowering. The results showed that $L c S V P$ s belonged to the MADS superfamily. $L c S V P$ relative expression levels in AMs of the early- and late-flowering cultivars showed decreasing trends with the transition from vegetative to reproductive growth. Under low temperature condition, relative expression levels of $L c S V P 1, L c S V P 2$, and $L c S V P 3$ in AMs or panicle primordia showed decreasing trends, whereas those in the AMs of the BR-treated trees remained at relatively high levels. Relative expression analysis of the litchi homolog, flowering locus $t$ 1 (LcFT1), showed that the BR-treated leaves had lower relative expression level than nontreated control leaves. The findings suggest that $L c S V P$ s act as repressors involved in flowering in natural conditions and the BR-regulated flowering.
\end{abstract}

Flowering plants undergo a transition from vegetative to reproductive growth. SVP, a MADS-domain transcription factor, was demonstrated to act as a repressor for flowering in arabidopsis (Hartmann et al., 2000) where it controls flowering by perceiving signals related to thermosensory, autonomous, and gibberelin (GA) pathways (Lee et al., 2007; Li et al., 2008). To maintain plants in a vegetative phase, SVP represses the expression of flowering locus $t(F T)$ and twin sister of FT (TSF) in the phloem and suppressor of overexpression of constans 1 $(\mathrm{SOCl})$ in the shoot apical meristem by directly binding to CArG boxes in FT and SOCl (Jang et al., 2009; Li et al., 2008). Although the role of SVP in flowering is well known in the model plant arabidopsis ( $\mathrm{Li}$ et al., 2008, 2010), little is known in evergreen woody plants.

Litchi is a Sapindaceae evergreen woody plant. It is also an important fruit tree that is widely cultivated in southeast Asia. Flowering in litchi is triggered by low temperatures and enhanced by drought in autumn and winter (Chen and Huang, 2005; Menzel and Simpson 1988). However, irregular flowering is a serious problem affecting litchi production ( $\mathrm{Lu}$ et al., 2014). During the past nearly 20 years, warm winters associated with global warming have resulted in poor litchi flowering.

Received for publication 5 Dec. 2017. Accepted for publication 22 Jan. 2018. This work was supported by the National Natural Science Foundation (project no. 31572080 and 31772249 ) and the China Agricultural Industry Project (project no. CARS-33).

${ }^{1}$ Corresponding author. E-mail: zhoubiyan@scau.edu.cn.
Hence, it is important to understand the genetics of flowering to find ways through its regulation. We have previously shown that reactive oxygen species (ROS) promotes flowering (Zhou et al., 2012). We then performed RNA-sequencing and identified a ROS-responsive $L c S V P$ in AMs of litchi and found that it might act as a floral repressor involved in the ROS-regulated flowering in litchi (Lu et al., 2017). However, the role of $L c S V P \mathrm{~s}$ in litchi flowering still needs further investigation.

Plant hormones are very important in flowering regulation (Campos-Rivero et al., 2017). The plant hormone signals are perceived and transmitted to the nuclear by series signal transduction components to regulate the expression of flowering related genes, such as constants (CO), flowering locus c (FLC), SVP, FT, and SOC1 (Conti, 2017; Li et al., 2010; Martínez et al., 2004). In litchi, abscisic acid was shown to promote flowering, whereas GAs inhibit flowering (Chen et al., 2014; Cui et al., 2013). Brassinosteroids are a group of polyhydroxylated plant steroid hormones that are ubiquitously present throughout the plant kingdom and play pivotal roles in plant growth and development with regulatory functions during cell elongation, cell division, vascular differentiation, and biotic and abiotic stress responses (Clouse and Sasse, 1998; Nolan et al., 2017). In arabidopsis, BR biosynthetic det2 mutants exhibited delayed flowering time (Li et al., 2010). In rice (Oryza sativa), SVPgroup MADS-box proteins are shown as negative regulators of BR response (Lee et al., 2008), demonstrating the role of $S V P$ in BR response in herbaceous plants. However, little is known about their roles in the flowering of evergreen woody litchi. 
In the present study, three litchi homologs $L c S V P 1, L c S V P 2$, and $L c S V P 3$ were cloned, and the bioinformatic analysis of the $L c S V P$ S was carried out to identify their molecular characteristics. Their expressions in the AM during the transition from vegetative to reproductive phase were studied, and BR treatment under inductive low temperature was performed to elucidate the role of $L c S V P \mathrm{~s}$ in litchi flowering.

\section{Materials and Methods}

Plant materials AND growth Conditions. Litchi trees were cultivated in the experimental orchard of South China Agricultural University (Guangzhou, China) (lat. 23 $3^{\circ} 40^{\prime \prime} \mathrm{N}$, long. $\left.113^{\circ} 21^{\prime} 18^{\prime \prime} \mathrm{E}\right)$. To determine the $L c S V P$ expression patterns, 12-year-old air-layered trees of early-flowering 'Sanyuehong' and late-flowering 'Nuomici' grown in open fields were selected. From Sept. 2015 to Feb. 2016, the trees were subjected to chilling by interrupted cold waves as the natural flowering inductive condition (Fig. 1). Samples were collected at five stages of floral formation according to Huang and Chen (2005). After the terminal shoots had just matured, the AMs of 'Sanyuehong' were collected on 15 Sept. 2015 as prefloral induction stage (PFId). Then AMs were collected on 14 Oct. as floral induction stage (FId). On 11 Nov. when panicle primordia of 'Sanyuehong' emerged and could be recognized as "whitish millets," the floral initiation stage (FIn), the panicle primordia were collected. After the FIn, panicle primordia continued to develop and were collected on 9 Dec. and 30 Dec. as the panicle developmental stage (PD). Similarly, samples of 'Nuomici' were collected on 15 Sept. as PFId stage, 11 Nov. and 9 Dec. as FId, 31 Jan. 2016 as FIn when the panicle primordia emerged, and 21 Feb. as PD as described by Lu et al. (2017). All the tissues were collected from three replicated trees and stored at $-80{ }^{\circ} \mathrm{C}$ for RNA extraction and gene expression analysis.

For the study of $L c S V P$ expression in different tissues, 12-year-old 'Nuomic' litchi trees subjected to winter chilling for floral induction were selected. Roots, stems, mature leaves, flowers, and buds were collected from three replicated trees from March to May, the flowering and fruiting season of litchi.

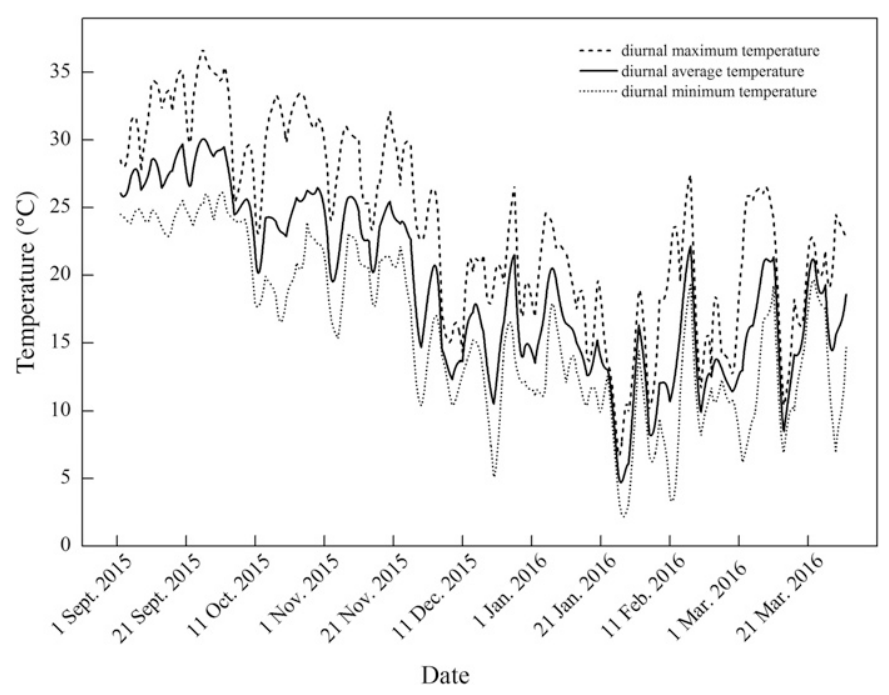

Fig. 1. Diurnal maximum temperature, diurnal average temperature, and diurnal minimum temperature changes in Autumn and Winter 2015-16.
Specifically, vegetative buds were picked up from new shoots, which could no longer produce floral buds at the season. All the tissues were frozen in liquid nitrogen and stored at $-80{ }^{\circ} \mathrm{C}$ for RNA extraction and gene expression analysis.

For BR treatment, 5-year-old air-layered 'Huaizhi' litchi trees were grown in $30-\mathrm{L}$ pots filled with loam, mushroom cinder, and coconut chaff (v:v:v, 3:1:1). Once the terminal shoots of the trees matured, 16 trees (1-1.5 m height, $\approx 100$ terminal shoots per tree) with the same phenological stage were selected for the experiment. The trees were transferred to a growth chamber made from polycarbonate sheet and grown under natural irradiance (transmittance of 92\%), a 12-h photoperiod, a relative humidity of $75 \%$ to $85 \%$, and $18 / 13{ }^{\circ} \mathrm{C}$ day/ night temperatures as inductive temperature (IT) for flowering. The trees were divided into two groups. One group was treated with $1 \mu \mathrm{M}$ 24-epibrassinolide (EBL), a member of the BR family, at $0,10,20,30$, and $40 \mathrm{~d}$ during IT treatment, and the other group was sprayed with water as the IT control at the same time. Either the EBL or water was applied as foliar spray to runoff $(\approx 200 \mathrm{~mL}$ per plant) using a pressure sprayer. Then AMs or panicle primordia were collected at $0 \mathrm{~d}$ (PFId), $30 \mathrm{~d}$ (FId), $60 \mathrm{~d}$ (FIn), and $80 \mathrm{~d}$ (PD) time points. Each sample was a mix of the AMs or panicle primordia from two trees, stored at $-80{ }^{\circ} \mathrm{C}$ for RNA extraction and $L c S V P$ expression analysis. At $0 \mathrm{~d}$ (PFId), $15 \mathrm{~d}$ (FId), $30 \mathrm{~d}$ (FId), $40 \mathrm{~d}$ (FId), $50 \mathrm{~d}$ (FId), $60 \mathrm{~d}$ (FIn), and $75 \mathrm{~d}$ (PD) time points, leaves of the terminal shoots from three replicated BR-treated or control trees were also collected and stored at $-80{ }^{\circ} \mathrm{C}$ for RNA extraction and LCFT1 expression analysis. At the 80-d time point, flowering conditions of the BR-treated and control trees were calculated from six replicated trees. Proportion of flowering trees was calculated as the proportion of the flowering trees to the total tested trees. Proportion of flowering terminal shoots was calculated as the proportion of the flowering terminal shoots to the total terminal shoots in one tree.

RNA Extraction. Total RNA was extracted using the Plant Total RNA Isolation Kit (Huayueyang, Beijing, China) according to the manufacturer's instruction and predigested with DNase I to eliminate the genomic DNA. The DNase-treated RNA concentration was determined using a spectrophotometer (NanoDrop 2000; Thermo Fisher Scientific, Wilmington, DE). The RNA integrity was visualized by $1.2 \%$ agarose gel electrophoresis.

Cloning and bioinformatic analysis of LcSVPs. The primers of $L c S V P 1-\mathrm{F} 1 / \mathrm{R} 1, L c S V P 2-\mathrm{F} 1 / \mathrm{R} 1$, and $L c S V P 3-\mathrm{F} 1 /$ R1 specific to $L c S V P 1, L c S V P 2$, and $L c S V P 3$, respectively (Table 1), were designed according to our transcriptome data (Lu et al., 2017). The PCR products were isolated and ligated into the pMD18-T vector for T/A cloning (Takara, Dalian, China), sequenced by Sangon Biotech Co. (Shanghai, China). The sequence of $L c S V P 1, L c S V P 1$, and $L c S V P 3$ was analyzed using BLAST program on the website of the National Center for Biotechnology Information (NCBI, Bethesda, MD). Conserved domain prediction was performed by CDD (NCBI). The sequences of the three $L c S V P$ s were aligned with those of longan (Dimocarpus longan), which belongs to the same family of Sapindaceae, and the model plant arabidopsis. The sequence alignment was performed using ClustalX 1.83 program (Thompson et al., 1997). The amino acid sequence was deduced by DNAMAN (Lynnon Corp., Vaudreuil, QC, Canada). The deduced amino acid sequences of the three LcSVPs and the eight top hit sequences from NCBI were used to construct 
Table 1. Primer sequences for cloning and quantitative real-time polymerase chain reaction (qRT-PCR).

\begin{tabular}{llll}
\hline Homology gene & \multicolumn{1}{c}{ Sequence $\mathrm{F}\left(5^{\prime} \rightarrow 3^{\prime}\right)$} & \multicolumn{1}{c}{ Sequence R $\left(5^{\prime} \rightarrow 3^{\prime}\right)$} & Amplicon size $(\mathrm{bp})$ \\
\hline$\beta$-actin & AGTTTGGTGATGTGGGGAC & TGGCTGAACCCGAGATGAT & Th1 \\
$L c S V P 1-\mathrm{F} 1 / \mathrm{R} 1$ & ATGGCGAGAGAGAAGATTCA & TTACCACCATAGGGTAAGCC & 682 \\
$L c S V P 2-\mathrm{F} 1 / \mathrm{R} 1$ & ATGACGAGACAGAAAATTGAGATC & TTAGCTAGGAAATGCAAACCCCAAC & 681 \\
$L c S V P 3-\mathrm{F} 1 / \mathrm{R} 1$ & ATGGCGAGAGAGAAGATCAA & TCAACTAGAGAAGGGAAGCCC & 100 \\
$L c S V P 1-\mathrm{F} 2 / \mathrm{R} 2$ & AGACAGCAAGTGGTGGAG & TGGTAACCGACTCTGATG & 171 \\
$L c S V P 2-\mathrm{F} 2 / \mathrm{R} 2$ & CCAAGGGTTGAATGTAGA & TTCCGTGTTCTTTGCCTC & 956 \\
$L c S V P 3-\mathrm{F} 2 / \mathrm{R} 2$ & TAAAACAAAGGGTGGACG & AGCAGATTCCGAAGACAA & 229 \\
\hline
\end{tabular}

$\mathrm{F}=$ forward; $\mathrm{R}=$ reverse.

a phylogenetic tree using the neighbor joining (NJ) method of the MEGA software [version 6.0 (Tamura et al., 2013)]. The secondary structure of LcSVPs was predicted by SOPMA (Geourjon and Deleage, 1995). Phyre version 2.0 (Kelley et al., 2015) was used to predict the $L c S V P$ s tertiary structure.

QUANTITATIVE REAL-TIME POLYMERASE CHAIN REACTION (RTPCR) ANALYSIS. First-strand cDNA was synthetized by using reverse transcriptase M-MLV [RNase H-(Takara)] from $1 \mu \mathrm{g}$ of extracted total RNA. As shown in Table 1, the quantitative realtime polymerase chain reaction (qRT-PCR) primers LcSVP1F2/R2 (length of amplicon $100 \mathrm{bp}$ ), LcSVP2-F2/R2 (length of amplicon $171 \mathrm{bp}$ ), LcSVP3-F2/R2 (length of amplicon $95 \mathrm{bp}$ ), and $L c F T-F / R$ (length of amplicon $229 \mathrm{bp}$ ) were designed using Primer 5.0 software (Premier Biosoft, Palo Alto, CA) and synthesized by Sangon Biotech Co. The litchi homolog $\beta$-actin (accession number HQ588865.1) was used as the reference gene because of its stable expression (Wei et al., 2012). The qRT-PCR was performed according to the method described by Yang et al. (2017) on a real-time PCR machine (CFX; Bio-Rad Laboratories, Hercules, CA). The qPCRs were run as follows: $95^{\circ} \mathrm{C}$ for $3 \mathrm{~min}$, followed by 40 cycles of $95^{\circ} \mathrm{C}$ for $15 \mathrm{~s}$, and $60{ }^{\circ} \mathrm{C}$ for $30 \mathrm{~s}$ in 96-well optical reaction plates (Bio-Rad Laboratories). Each qRT-PCR analysis was performed in triplicate. The transcript quantification of the genes was performed in relation to Actin and they were calculated by $2^{-\Delta \Delta C T}$ method (Livak and Schmittgen, 2001).

Statistical ANALysis. Reported data of flowering condition are the means of the six replicates, and those of the qRT-PCR are the means of the three replicates. Statistical analyses were performed by variance using SPSS (version 19.0; IBM Corp., Armonk, NY). The differences among treatment means were evaluated by using Duncan's multiple range test at a $0.05 P$ level, and those between the treatment and the control were evaluated by using Student's test at a $0.05 P$ level. SE was calculated by using Microsoft Excel 2010 (Microsoft, Redmond, WA).

\section{Results}

Cloning and characterization of LCSVPs. We identified a 684-bp-long open reading frame (ORF) of LcSVP1 with 227 deduced amino acids, a 642-bp-long ORF of LcSVP2 with 213 deduced amino acids, and a 681-bp-long ORF of LcSVP3 with 226 deduced amino acids (Supplemental Fig. 1). NCBI BLASTp results showed that $L c S V P 1, L c S V P 2$, and $L c S V P 3$ belonged to the MADS superfamily (Supplemental Fig. 2). Alignment of predicted protein sequences showed that the MADS box and K box among the SVPS share high similarity (Fig. 2). The predicted secondary structure indicated that

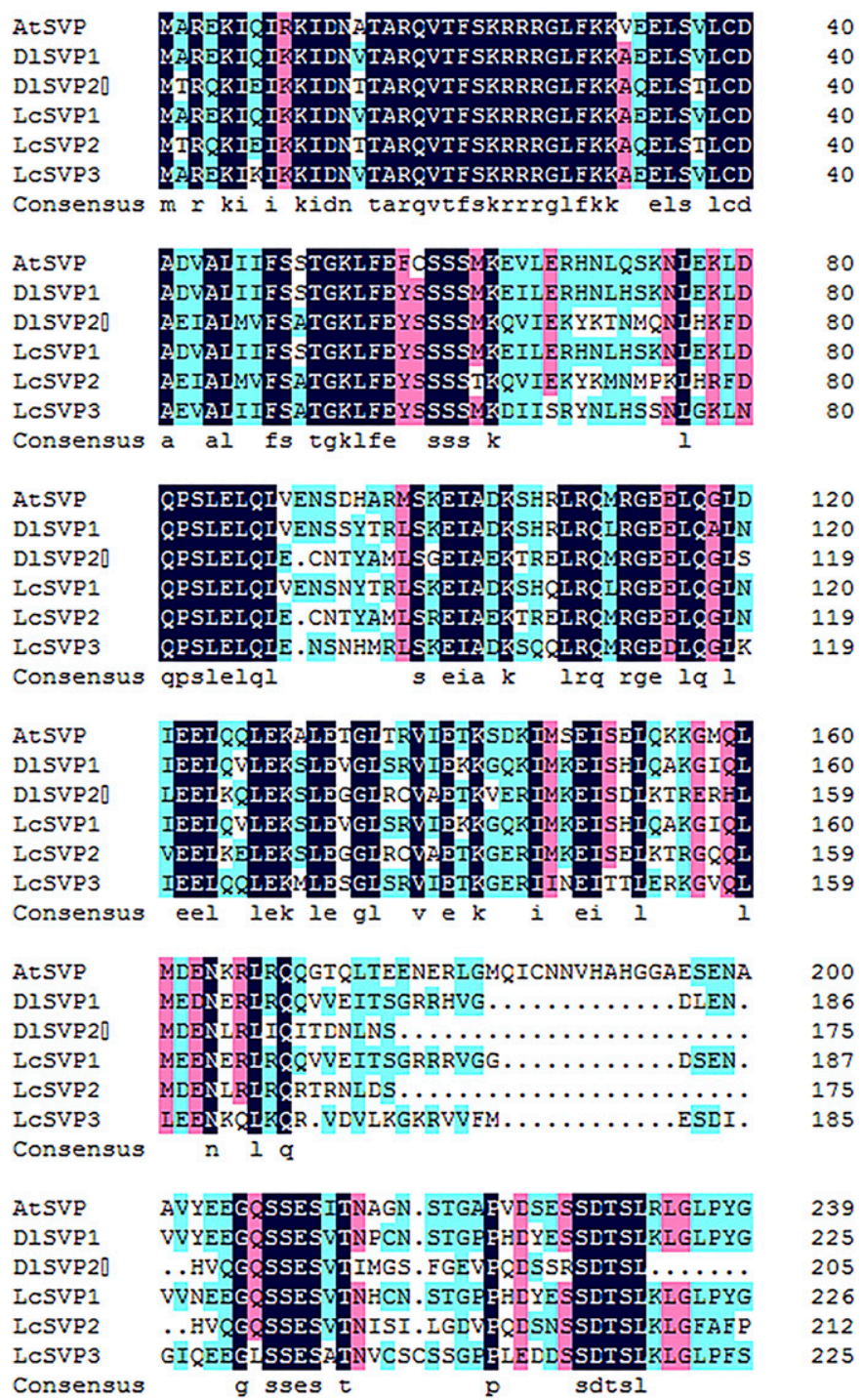

Fig. 2. Alignment of predicted protein sequences of the short vegetative phase (SVP) homologs LcSVP1, LcSVP2, LcSVP3, DlSVP1, DlSVP1, and AtSVP (Arabidopsis thaliana: AtSVP; Litchi chinensis: LcSVP1, LcSVP2, LcSVP1; Dimocarpus longan: DlSVP1, DlSVP1).

LcSVP1 contained 119 alpha helices, 16 beta turns, and 92 random coils. $L c S V P 2$ contained 117 alpha helices, 16 beta turns, and 80 random coils. LcSVP3 contained 120 alpha helices, 16 beta turns, and 90 random coils (Supplemental Fig. 3). Tertiary structure prediction indicated that the structures of LcSVP1 and LcSVP3 are similar to each other, whereas that of 
LCSVP 2 is quite different from the other two (Supplemental Fig. 4). Phylogenetic analysis indicated that the three genes belonged to two branches. LcSVPI and $L c S V P 3$ were clustered in one branch, in which the LcSPVI shared $100 \%$ identity with longan SVP 1 (DlSVP 1). LcSVP2 and $D l S V P 2$ which shared $100 \%$ identity were clustered in another branch (Fig. 3).

LCSVPS EXPRESSION PATTERNS IN DIFFERENT TISSUES. To determine the transcriptional expression patterns of $L c S V P \mathrm{~s}$ in the vegetative and reproductive tissues, roots, stems, flowers, leaves, vegetative buds, and fruit of 'Nuomici' were separately collected. Expression levels of $L c S V P 1, L c S V P 2$, and $L c S V P 3$ in the specific tissues were determined by using qRT-PCR. As shown in Fig. 4, $L C S V P 1$ is strongly expressed in vegetative buds, but less expressed in roots, stems, leaves, flowers, and fruit. The relative expression of LCSVP1 in buds was 57.2- and 13.3-fold higher than that in roots and fruit, respectively. $L c S V P 2$ was strongly expressed in vegetative buds and leaves, but less expressed in roots, stems, flowers, and fruit. Relative expression level of $L c S V P 1$ in leaves and buds was 9.9- and 9.5-fold higher than that in flowers, respectively. No transcription of $L C S V P 2$ could be determined in fruit tissues. Relative expression of $L c S V P 3$ in buds was significantly higher than that in roots, stems, leaves, flowers, and fruit. On the whole, LcSVPs were strongly expressed in vegetative buds, but less expressed in roots, flowers, and fruit, indicating that $L c S V P$ s expression was suppressed in roots and the reproductive tissues of flowers and fruit.

LCSVPS EXPRESSION PATTERNS IN DIFFERENT CULTIVARS UNDER NATURAL FLOWERING INDUCTIVE CONDITIONS. To find out whether the $L c S V P$ s transcription was suppressed during the transition from vegetative to reproductive growth, the AMs or panicle primordia were collected from the early-flowering 'Sanyuehong' and the late-flowering 'Nuomici' in autumn and winter, during which the temperature decreased, and the trees were subjected to chilling by interrupted cold waves (Fig. 1). The litchi trees were therefore subjected to natural chilling conditions for floral induction. The transformation from vegetative buds to reproductive floral buds happened in this period. On 11 Sept. 2015, panicle prmirodia emerged in the early flowering 'Sanyuehong' (Fig. 5A), whereas on 31 Jan. 2016, panicle prmirodia emerged in the late-flowering 'Nuomici' (Fig. 5B). Relative expression levels of $L c S V P 1, L c S V P 2$, and $L c S V P 3$ in these tissues were determined from PFId to PD. The results indicated that relative expression levels of these three $L c S V P \mathrm{~s}$ in both cultivars showed decreasing trends with the transition from vegetative to reproductive growth (Fig. 5C).

LCSVPS EXPRESSION PATTERNS UNDER BR-REGULATED FLOWERING CONDITION. To further study the $L c S V P$ s expression 
A
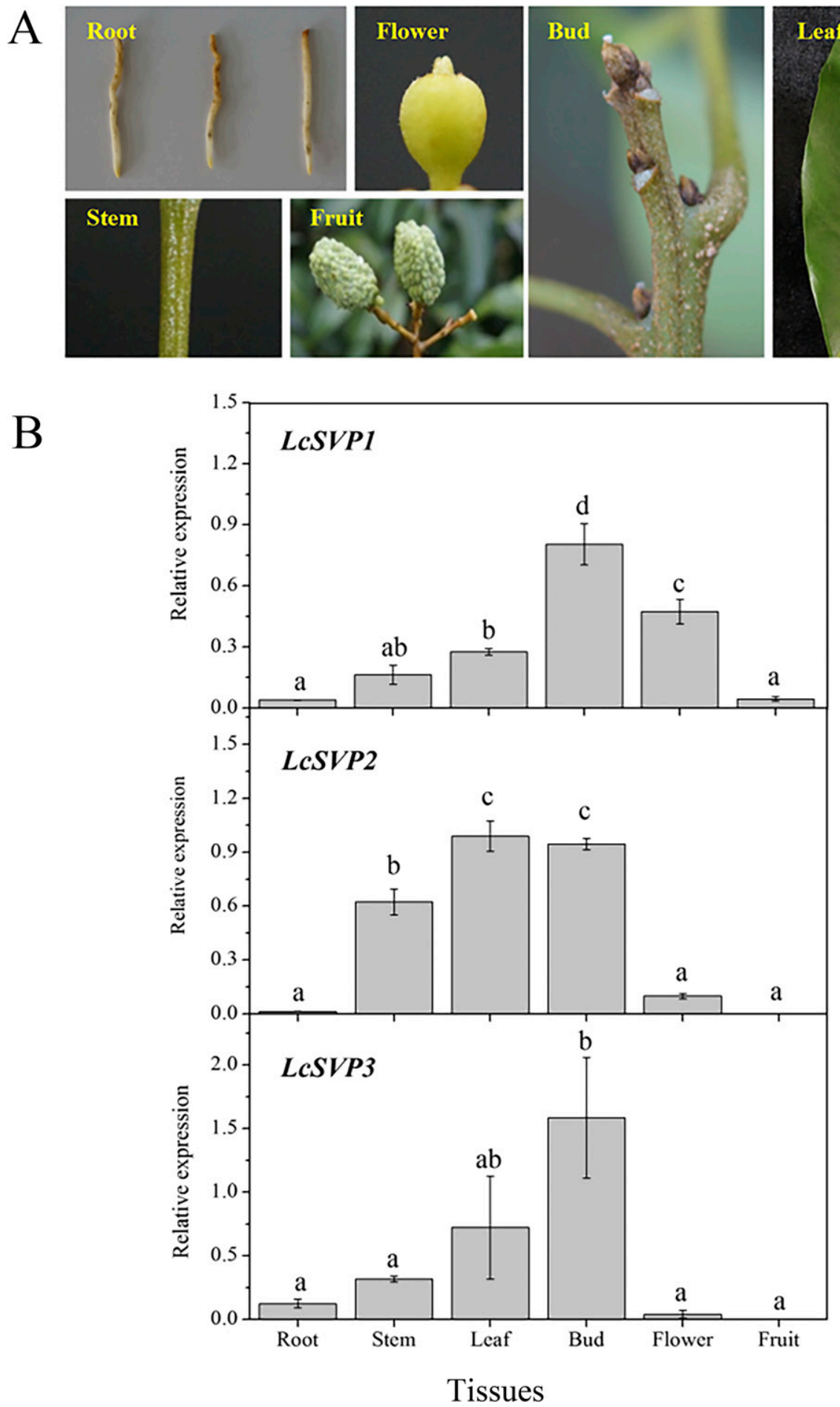

Fig. 4. Expression patterns of the short vegetative phase (SVP) homologs $L c S V P 1, L c S V P 2$, and $L c S V P 3$ in different tissues of 'Nuomici' litchi. (A) Images of the roots, stems, flowers, fruit, buds, and leaves. (B) Relative expression of the $L c S V P 1, L c S V P 2$, and $L c S V P 3$. Data are means of three replicates and the bars represent SES. Different lowercase letters above column means indicate significance at $P<0.05$ according to Duncan's multiple rage test using SPSS (version 19.0; IBM Corp.).

patterns by BR treatment, potted trees were sprayed with BR or water in a growth chamber under floral IT. After $80 \mathrm{~d}$ of treatment, flowering phenotype of the trees was determined. As shown in Table 2, the proportion of flowering trees of the BR treatment was lower than that of the control, and the proportion of flowering terminal shoots of the BR-treated trees was

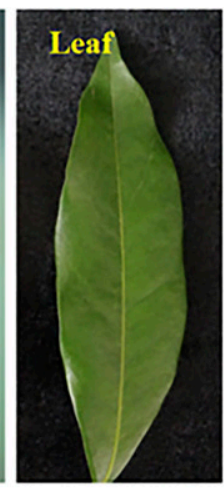

significantly lower than that of the control one. The results suggested that BR inhibited flowering of litchi under IT conditions. We further determined the $L c S V P$ relative expression levels. As shown in Fig. 6, on the whole, relative expression levels of $L c S V P 1, L c S V P 2$, and $L c S V P 3$ in AMs or panicle primordia of the control trees showed decreasing trends, whereas those of the BRtreated trees remained at relatively high levels. Relative expression analysis of LcFT1 showed that the BR-treated leaves had lower levels than the control ones. For example, at 40- and 60-d time points, relative expression of LcFT1 in control leaves was 42.9- and 27.3-fold higher than that in BR-treated leaves, respectively (Fig. 7).

\section{Discussion}

Flowering is a genetically controlled process during the transition from vegetative to reproductive development. Stress is simply defined as a condition in which the vegetative growth of plants is suppressed (Takeno, 2012). Many of the environmental conditions inducing flowering can be considered as stress conditions. The stressed plants do not need to wait for the arrival of a season when photoperiodic conditions are suitable for flowering, and such precocious flowering may be important for species preservation (Takeno, 2012). Under stressful conditions, ROS accumulates and functions as signals regulating plant growth and development (Ahanger et al., 2017). Flowering in litchi is triggered by low temperatures and enhanced by drought stress in autumn and winter (Chen and Huang, 2005; Menzel and Simpson, 1988). These two inductive factors can be regarded as stress conditions that can suppress vegetative growth of litchi. We have previously shown that ROS promotes flowering (Zhou et al., 2012), and identified ROS-responsive LCSVP1 in AMs of litchi. Our previous data showed that $L c S V P 1$ might be involved in the ROScontrolled floral transition (Lu et al., 2017). We hypothesized that the $L c S V P$ s might act as repressors in litchi flowering. Hence, in the present study, we clone the $S V P$ homologs identified from our RNA-seq data set (Lu et al., 2014, 2017). We found that $L c S V P 1$, $L c S V P 2$, and $L c S V P 3$ belonged to the MADS superfamily. 

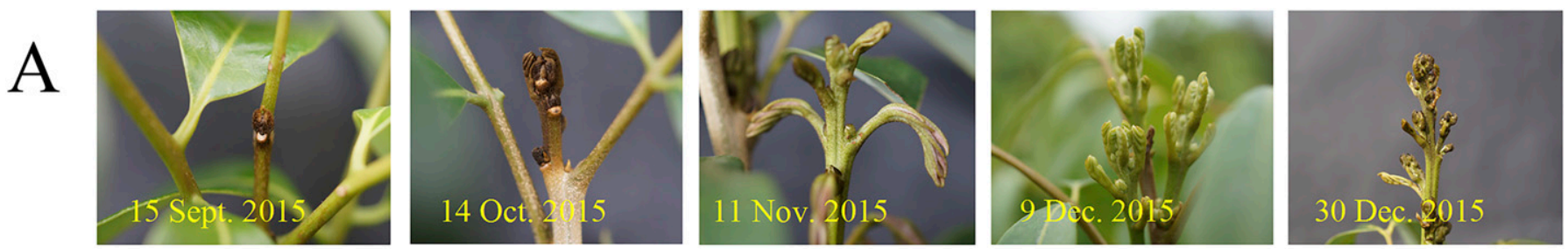

$\mathrm{B}$
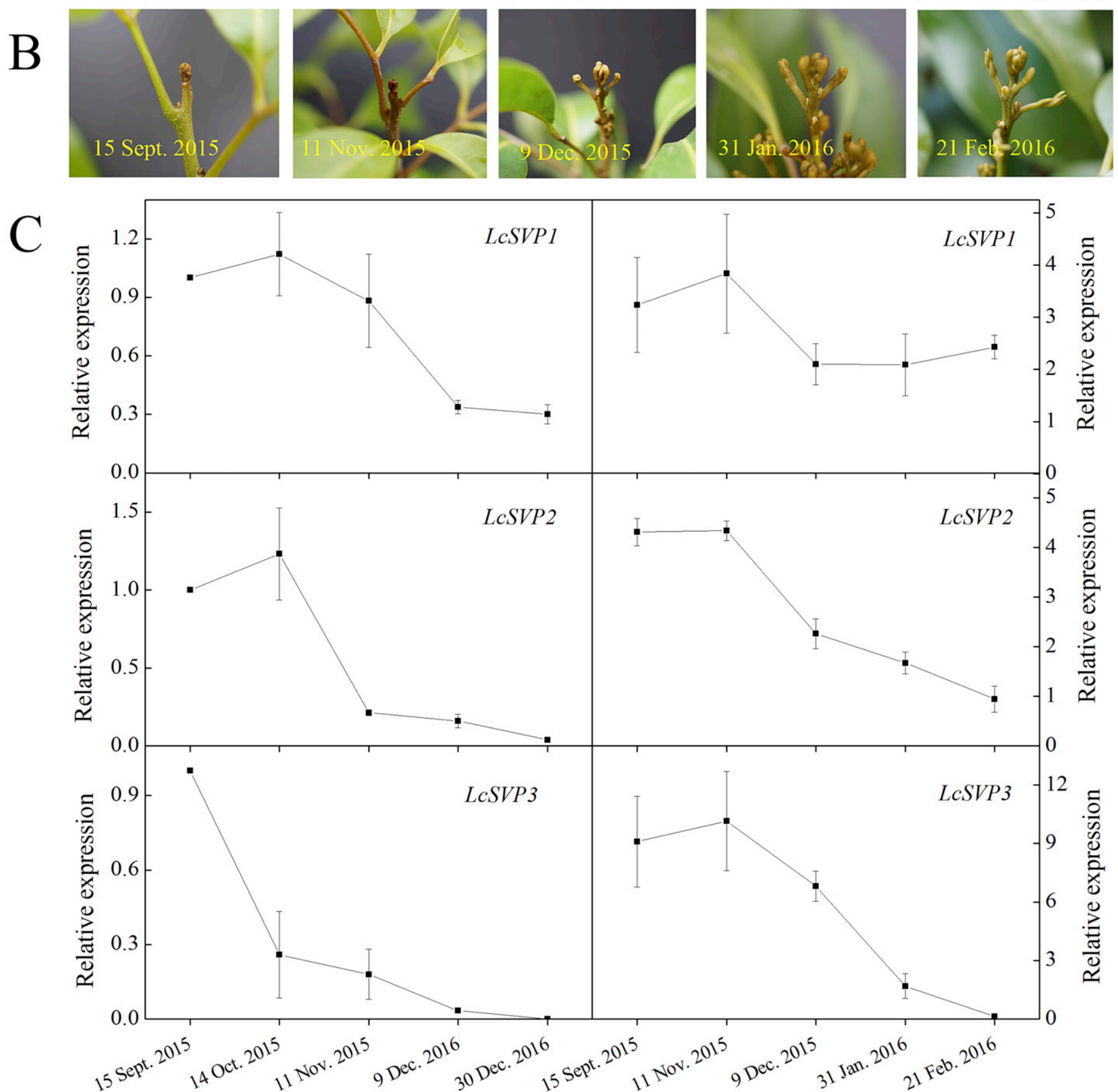

Sampling date

Sampling date

Fig. 5. Morphology of the buds in 'Sanyuehong' (A) and 'Nuomici' (B) litchi from the prefloral induction stage (PFId) to the panicle developmental stage (PD), and expression patterns of the short vegetative phase (SVP) homologs $L c S V P 1, L c S V P 2$, and $L c S V P 3$ in apical meristems (AMs) or panicle primordia of 'Sanyuehong' and 'Nuomici' in the nature-controlled flowering condition $(\mathbf{C})$. Trees of the early flowering 'Sanyuehong' and the late-flowering 'Nuomici' grown in open fields were selected. AMs or panicle primordia of 'Sanyuehong' were collected on 15 Sept. 2015 as PFId, 14 Oct. as floral induction stage (FId), Nov. 11 as floral initiation stage (FIn), and 9 Dec. and 30 Dec. as the PD. Those of the 'Nuomici' were collected on 15 Sept. as PFId stage, 11 Nov. and 9 Dec. as FId, 31 Jan. 2016 as FIn, and 21 Feb. as PD. Data are means of three replicates and the bars represent sEs. 
Table 2. Effects of brassinosteroid (BR) on the flowering of 'Huaizhi' litchi trees under inductive temperatures $(n=6)$.

\begin{tabular}{lcc}
\hline Treatments & Proportion of flowering trees $(\%)^{\mathrm{z}}$ & Proportion of flowering terminal shoots [mean \pm SE $(\%)]^{\mathrm{y}}$ \\
\hline BR & 33.3 & $1.48 \pm 1.13$ \\
Control & 100 & $29.30 \pm 11.69^{*}$
\end{tabular}

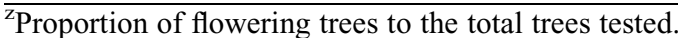

${ }^{\mathrm{y}}$ Proportion of flowering terminal shoots to the total terminal shoots in one tree. Means follows by asterisks are significant different at $P \leq 0.05$.
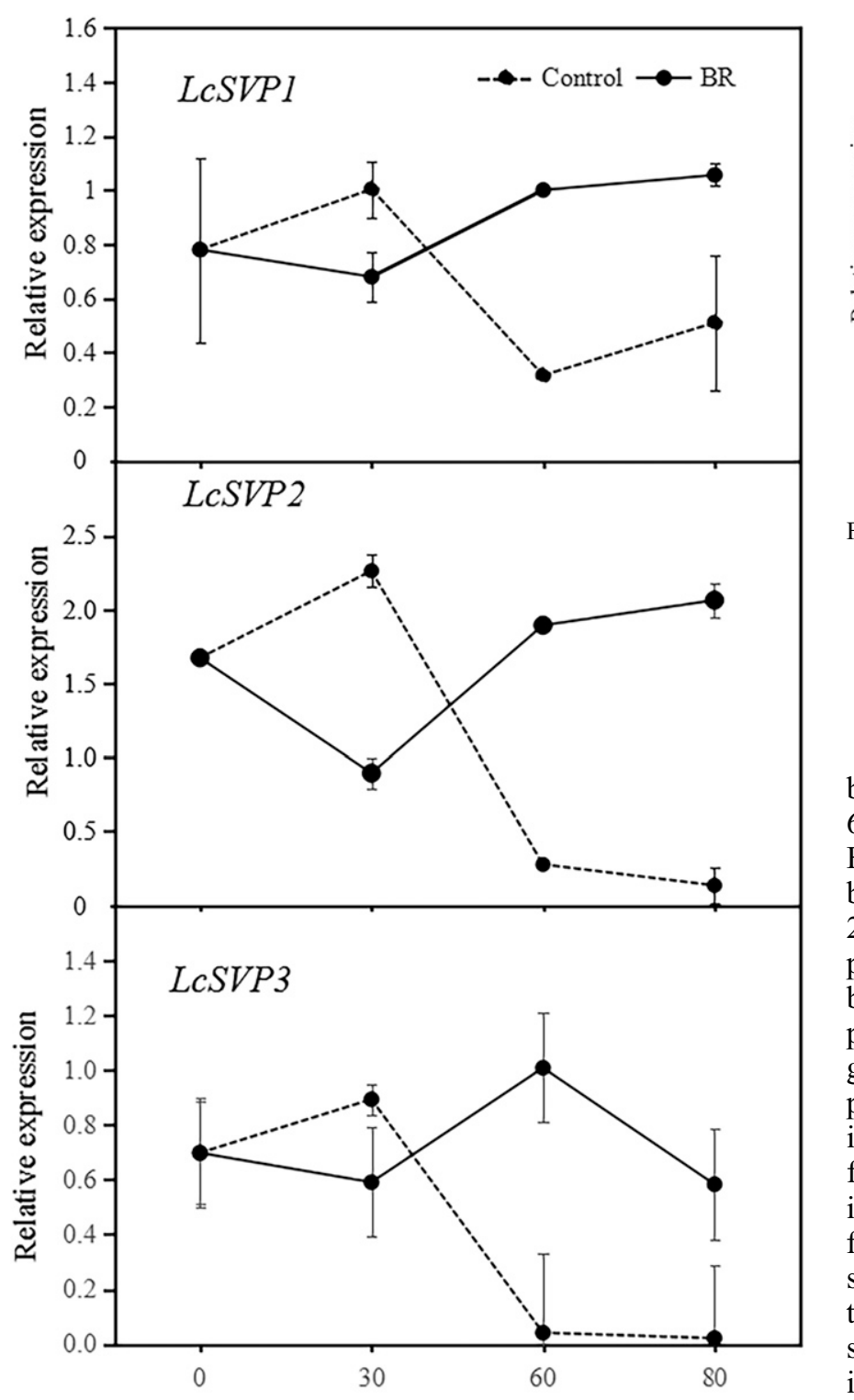

Time of treatment (d)

Fig. 6. Expression patterns of the short vegetative phase (SVP) homologs $L c S V P 1, L c S V P 2$, and $L c S V P 3$ in apical meristems (AMs) or panicle primordia of the brassinosteroid-treated litchi trees under controlled inductive temperature (IT) conditions. Potted litchi trees were transferred to a growth chamber at $18 / 13{ }^{\circ} \mathrm{C}$ [day/night temperature $(12 / 12 \mathrm{~h}$ day/night)] as IT for flowering. The trees were sprayed with $1 \mu \mathrm{M}$ 24-epibrassinolide (EBL), or water as control at $0,10,20,30$, and $40 \mathrm{~d}$ of IT treatment. Data are means of three replicates and the bars represent SES.

MADS-domain transcription factors have been identified in plants and were proved to be involved in flowering (Masiero et al., 2011). MADS-domain factors activate or repress transcription by directly binding to short sequences called CArG

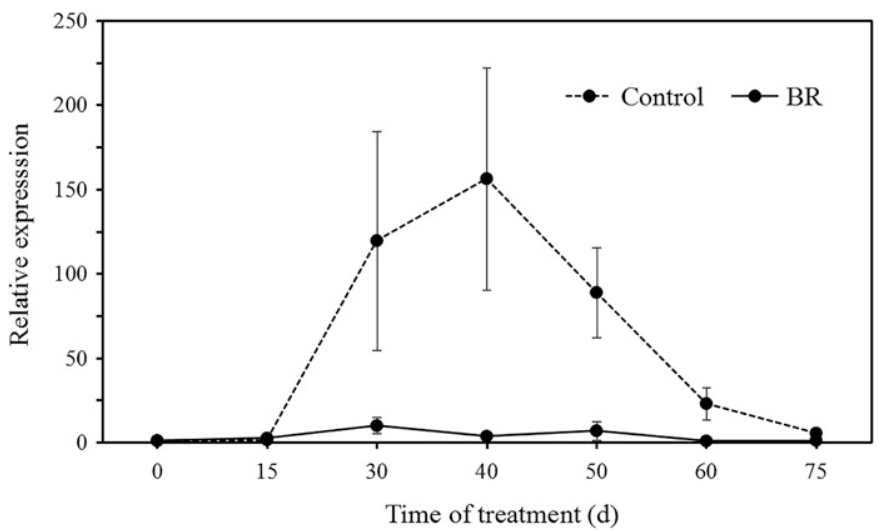

Fig. 7. Expression patterns of the litchi homolog flowering locus 1 ( LcFTl) in leaves of the brassinosteroid-treated trees under controlled inductive temperature (IT) condition. Trees were transferred to a growth chamber at $18 / 1{ }^{\circ} \mathrm{C}$ [day/night temperature $(12 / 12 \mathrm{~h}$ day/night)] as IT for flowering. The trees were sprayed with $1 \mu \mathrm{M} 24$-epibrassinolide (EBL), or water as control at 0,10 , 20,30 , and $40 \mathrm{~d}$ of IT treatment. Data are means of three replicates and the bars represent SES.

boxes that correspond to a 10 nucleotide sequence, $\mathrm{CC}(\mathrm{A} / \mathrm{T})$ $6 \mathrm{GG}$, present in the regulatory sequences of target genes (de Folter and Angenent, 2006). In arabidopsis, $S V P$ binds to $\mathrm{CArG}$ boxes in $F T$ and $S O C l$ and acts as a floral repressor (Jang et al., 2009; Li et al., 2008). In kiwifruit (Actinidia sp.), the woody perennial vines, $S V P$-like genes may have distinct roles during bud dormancy and flowering (Wu et al., 2012). In woody tree peony (Paeonia suffruticosa), PsSVP can promote vegetative growth and suppress flowering (Wang et al., 2014). In the present study, we found that the $L c S V P$ s were strongly expressed in vegetative buds and leaves, but least expressed in flowers and fruit, indicating that $L c S V P$ s relative expression was suppressed in the reproductive tissues. Also, those levels in both the early flowering 'Sanyuehong' and the late-flowering 'Nuomici' showed decreasing trends with the transition from vegetative to reproductive growth under the floral inductive condition, suggesting that $L c S V P$ s might act as repressors in litchi flowering, similar to those of the arabidopsis, kiwifruit, and tree peony (Jang et al., 2009; Wang et al., 2014; Wu et al., 2012).

To further study the role of $L c S V P$ s in litchi flowering, their relative expression levels were determined under hormonal regulation. We sprayed potted litchi trees with the plant steroid hormone 24-EBL, a member of the BR family, and placed them under flowering ITs $\left(18 / 13{ }^{\circ} \mathrm{C}\right)$ in the growth chamber. To our surprise, BR-inhibited flowering under inductive low temperature treatment, which is contrary from what were reported in herbaceous rice and arabidopsis (Lee et al., 2008; Li et al., 2010). BRs are regarded as growth-promoting hormones (Khripach et al., 2000). It has been proved that they can promote biosynthesis of GA, another growth-promoting hormone (Unterholzner et al., 2015). BR-regulated transcription factor BES1 binds to a regulatory element in promoters of GA 
biosynthesis genes to control their relative expression in a BRinduced manner (Unterholzner et al., 2015). GAs promote flowering in arabidopsis (Blazquez et al. 1998). In contrast, GAs inhibit flowering in litchi (Chen et al., 2014), which is evidenced by the fact that exogenous application of paclobutrazol, a GA biosynthesis inhibitor, promotes flowering (Ray and Ruby, 2004) and is a practical method for litchi production. Similar results were reported in citrus (Citrus sp.), a group of evergreen woody trees (Goldschmidt et al., 1997). Based on the contrasting effects of GA on flowering mechanism between the evergreen woody tree litchi and the herbaceous arabidopsis, it is speculated that BR has diverse effects on flowering of the two species. In our present study, reduced flowering was observed in BR-treated trees, of which relative expression levels of $L c S V P$ s remained higher than the control trees. In addition, relative expression level of LcFT1 whose encoding protein is homologous to the arabidopsis florigen (Corbesier et al., 2007; Ding et al., 2015; Yang et al., 2007) was lower in the BR-treated leaves than that in the control ones. In litchi, two FT homologue genes LcFT1 and LcFT2 were identified. However, low temperature can only induce $L c F T 1$ expression in leaves. Overexpression of $L c F T 1$ in tobacco and arabidopsis induces precocious flowering (Ding et al., 2015). It is suggested that LcFT1 expression is positively correlated with litchi flowering. Hence, in the present study, we determined the relative expression of $L c F T 1$. In accordance with the poor flowering in the BR-treated trees, a lower expression of LcFT1 in the BR-treated trees was found compared with the control trees. The expression of $L c S V P$ s under BR regulation further suggested that $L c S V P$ s might act as floral repressors through regulating the transcription of $L c F T 1$ expression. Future study should be focused on the relationship between LcSVPs and LcFT1.

In conclusion, we cloned three $L c S V P$ genes, and they were strongly expressed in buds, but less expressed in flowers and fruit. $L c S V P$ relative expression levels in both of the early- and late-flowering cultivars showed decreasing trends with the transition from vegetative to reproductive growth. Relative expression levels of $L c S V P 1, L c S V P 2$, and $L c S V P 3$ in AMs or panicle primordia of the control trees showed decreasing trends with the transition from vegetative to reproductive growth, whereas those in AMs of the BR-treated trees remained at relatively high levels. $L c S V P$ s might act as floral repressors involved in flowering in natural conditions and the BRregulated flowering.

\section{Literature Cited}

Ahanger, M.A., N.A. Akram, M. Ashraf, M.N. Alyemeni, L. Wijaya, and P. Ahmad. 2017. Signal transduction and biotechnology in response to environmental stresses. Biol. Plant. 61:401-416.

Blazquez, M.A., R. Green, O. Nilsson, M.R. Sussman, and D. Weigel. 1998. Gibberellins promote flowering of arabidopsis by activating the $L E A F Y$ promoter. Plant Cell 10:791-800.

Campos-Rivero, G., P. Osorio-Montalvo, R. Sanchez-Borges, R. UsCamas, F. Duarte-Ake, and C. De-la-Pena. 2017. Plant hormone signaling in flowering: An epigenetic point of view. J. Plant Physiol. 214:16-27.

Cui, Z., B. Zhou, Z. Zhang, and Z. Hu. 2013. Abscisic acid promotes flowering and enhances LcAP1 expression in Litchi chinensis Sonn. S. Afr. J. Bot. 88:76-79.

Chen, H.B. and H.B. Huang. 2005. Low temperature requirements for floral induction in lychee. Acta Hort. 665:195-202.
Chen, P.A., C.L. Lee, S.F. Roan, and I.Z. Chen. 2014. Effects of $\mathrm{GA}_{3}$ application on the inflorescence and yield of 'Yu Her Pau' litchi. Scientia Hort. 171:45-50.

Conti, L. 2017. Hormonal control of the floral transition: Can one catch them all? Dev. Biol. 430:288-301.

Clouse, S.D. and J.M. Sasse. 1998. Brassinosteroids: Essential regulators of plant growth and development. Annu. Rev. Plant Physiol. Plant Mol. Biol. 49:427-451.

Corbesier, L., C. Vincent, S.H. Jang, F. Fornara, Q. Fan, I. Searle, A. Giakountis, S. Farrona, L. Gissot, C. Turnbull, and G. Coupland. 2007. FT protein movement contributes to long-distance signaling in floral induction of Arabidopsis. Science 316:1030-1033.

de Folter, S. and G.C. Angenent. 2006. Trans meets cis in MADS science. Trends Plant Sci. 11:224-231.

Ding, F., S.W. Zhang, H.B. Chen, Z.X. Su, R. Zhang, Q.S. Xiao, and H.L. Li. 2015. Promoter difference of LcFT1 is a leading cause of natural variation of flowering timing in different litchi cultivars (Litchi chinensis Sonn.). Plant Sci. 241:128-137.

Geourjon, C. and G. Deleage. 1995. SOPMA: Significant improvements in protein secondary structure prediction by consensus prediction from multiple alignments. Bioinformatics 11:681-684.

Goldschmidt, E.E., M. Tamim, and R. Goren. 1997. Gibberellins and flowering in citrus and other fruit trees: A critical analysis. Acta Hort. 463:201-208.

Hartmann, U., S. Hohmann, K. Nettesheim, E. Wisman, H. Saedler, and P. Huijser. 2000. Molecular cloning of SVP: A negative regulator of the floral transition in Arabidopsis. Plant J. 21:351-360.

Huang, H.B. and H.B. Chen. 2005. A phase approach towards floral formation in lychee. Acta Hort. 665:185-194.

Jang, S., S. Torti, and G. Coupland. 2009. Genetic and spatial interactions between FT, TSF and SVP during the early stages of floral induction in Arabidopsis. Plant J. 60:614-625.

Kelley, L.A., S. Mezulis, C.M. Yates, M.N. Wass, and M.J.E. Sternberg. 2015. The Phyre2 web portal for protein modeling, prediction and analysis. Nat. Protoc. 10:845-858.

Khripach, V.A., V.N. Zhabinskii, and A.E. de Groot. 2000. Twenty years of brassinosteroids: Steroidal plant hormones warrant better crops for the XXI century. Ann. Bot. 86:441-447.

Lee, J.H., S.J. Yoo, S.H. Park, I. Hwang, J.S. Lee, and J.H. Ahn. 2007. Role of SVP in the control of flowering time by ambient temperature in Arabidopsis. Genes Dev. 21:397-402.

Lee, S., S.C. Choi, and G. An. 2008. Rice SVP-group MADS-box proteins, OsMADS22 and OsMADS55, are negative regulators of brassinosteroid responses. Plant J. 54:93-105.

Li, D., C. Liu, L. Shen, Y. Wu, H. Chen, M. Robertson, C.A. Helliwell, T. Ito, E. Meyerowitz, and H. Yu. 2008. A repressor complex governs the integration of flowering signals in Arabidopsis. Dev. Cell 15:110-120.

Li, J.H., Y.H. Li, S.Y. Chen, and L.Z. An. 2010. Involvement of brassinosteroid signals in the floral-induction network of Arabidopsis. J. Expt. Bot. 61:4221-4230.

Livak, K.J. and T.D. Schmittgen. 2001. Analysis of relative gene expression data using real-time quantitative PCR and the $2^{-\Delta \Delta C T}$ method. Methods 25:402-408.

Lu, X., H. Kim, S. Zhong, H. Chen, Z. Hu, and B. Zhou. 2014. De novo transcriptome assembly for rudimentary leaves in Litchi chinesis Sonn. and identification of differentially expressed genes in response to reactive oxygen species. BMC Genomics 15:805.

Lu, X., J. Li, H. Chen, J. Hu, P. Liu, and B. Zhou. 2017. RNA-seq analysis of apical meristem reveals integrative regulatory network of ROS and chilling potentially related to flowering in Litchi chinensis. Sci. Rpt. 7:10183.

Martínez, C., E. Pons, G. Prats, and J. León. 2004. Salicylic acid regulated flowering time and link defence responses and reproductive development. Plant J. 37:209-217.

Masiero, S., L. Colombo, P.E. Grini, A. Schnittger, and M.M. Kater. 2011. The emerging importance of type I MADS box transcription factors for plant reproduction. Plant Cell 23:865-872. 
Menzel, C.M. and D.X. Simpson. 1988. Effect of temperature on growth and flowering of litchi (Litchi chinensis Sonn.) cultivars. J. Hort. Sci. 63:349-360.

Nolan, T., J. Chen, and Y. Yin. 2017. Cross-talk of brassinosteroid signaling in controlling growth and stress responses. Biochem. J. 474:2641-2661.

Ray, P.K. and R. Ruby. 2004. Effect of paclobutrazol on flowering in "China" litchi (Litchi chinensis Sonn.). Indian J. Plant. Physiol. 9:208-211.

Takeno, K. 2012. Stress-induced flowering, p. 331-345. In: P. Ahmad and M.N.V. Prasad (eds.). Abiotic stress responses in plants. Springer, New York, NY.

Tamura, K., G. Stecher, D. Peterson, A. Filipski, and S. Kumar. 2013. MEGA6: Molecular evolutionary genetics analysis version 6.0. Mol. Biol. Evol. 30:2725-2729.

Thompson, J.D., T.J. Gibson, F. Plewniak, F. Jeanmougin, and D.G. Higgins. 1997. The CLUSTAL_X windows interface: Flexible strategies for multiple sequence alignment aided by quality analysis tools. Nucl. Acids Res. 25:4876-4882.

Unterholzner, S.J., W. Rozhon, M. Papacek, J. Ciomas, T. Lange, K.G. Kugle, K.F. Mayer, T. Sieberer, and B. Poppenberger. 2015. Brassinosteroids are master regulators of gibberellin biosynthesis in Arabidopsis. Plant Cell 27:2261-2272.
Wang, S., J. Xue, N. Ahmadi, P. Holloway, F. Zhu, X. Ren, and X. Zhang. 2014. Molecular characterization and expression patterns of PSSVP genes reveal distinct roles in flower bud abortion and flowering in tree peony (Paeonia suffruticosa). Can. J. Plant Sci. 94:1181-1193.

Wei, Y.Z., B. Lai, F.C. Hu, X.J. Li, G.B. Hu, and H.C. Wang. 2012. Cloning and stability analysis of reference genes for expression studies by quantitative real-time PCR in litchi. J. South China Agr. Univ. 33:303-306.

Wu, R.M., E.F. Walton, A.C. Richardson, M. Wood, R. Hellens, and E. Varkonyi-Gasic. 2012. Conservation and divergence of four kiwifruit SVP-like MADS-box genes suggest distinct roles in kiwifruit bud dormancy and flowering. J. Expt. Bot. 63:797-807.

Yang, H.F., X.Y. Lu, H.B. Chen, C.C. Wang, and B.Y. Zhou. 2017. Low temperature-induced leaf senescence and the expression of senescence-related genes in the panicles of Litchi chinensis. Biol. Plant. 61:315-322.

Yang, Y.J., J. Klejnot, X.H. Yu, X.M. Liu, and C.T. Lin. 2007. Florigen (II): It is a mobile protein. J. Integr. Plant Biol. 49:1665-1669.

Zhou, B., N. Li, Z. Zhang, X. Huang, H. Chen, Z. Hu, X. Pang, W. Liu, and Y. Lu. 2012. Hydrogen peroxide and nitric oxide promote reproductive growth in Litchi chinensis. Biol. Plant. $56: 321-329$. 


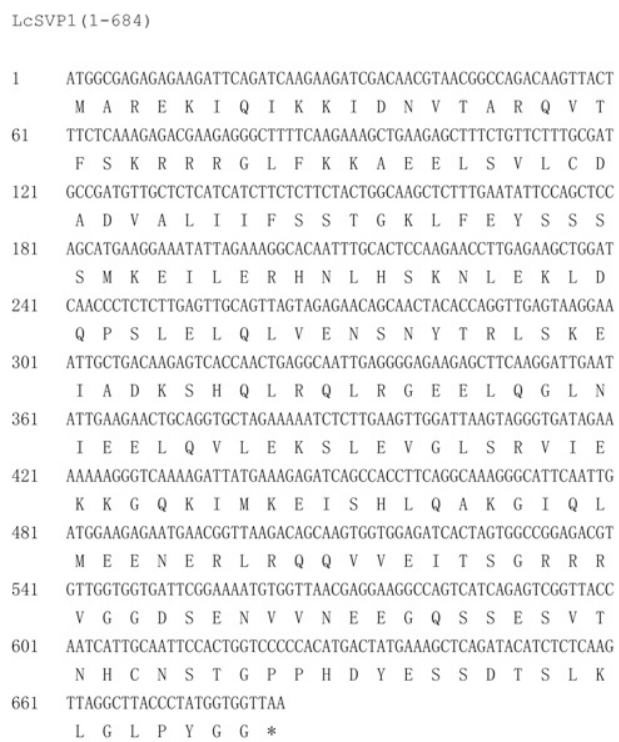

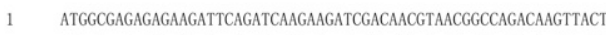

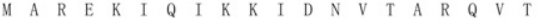
61 TTCTCAAAGAGACGAAGAGGGCTTTTCAAGAAAGCTGAAGAGCTTTCTGTTCTTTGCGAT

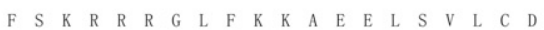
121 GCCGATGTTGCTCTCATCATCTTCTCTTCTACTGGCAAGCTCTTTGAATATTCCAGCTCC

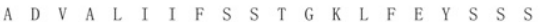
181 AGCATGAaGgaAATATTAGAAAGGCACAATTTGCACTCCAAGAACCTTGAGAaGCTGGAT

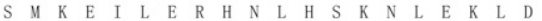
241 CAACCCTCTCTTGAGTTGCAGTTAGTAGAGAACAGCAACTACACCAGGTTGAGTAAGGAA Q $\begin{array}{llllllllllllllllllll} & P & S & L & E & L & Q & L & V & E & N & S & N & Y & T & R & L & S & K & E\end{array}$ 301 ATTGCTGACAAGAGTCACCAACTGAGGCAATTGAGGGGAGAAGAGCTTCAAGGATTGAAT

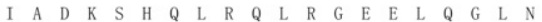
361 ATTGAAGAACTGCAGGTGCTAGAAAAATCTCTTGAAGTTGGATTAAGTAGGGTGATAGAA

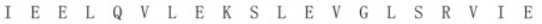
421 AAAAAGGGTCAAAAGATTATGAAAGAGATCAGCCACCTTCAGGCAAAGGGCATTCAATTG $\begin{array}{lllllllllllllllllllll}K & K & G & Q & K & I & M & K & E & I & S & H & L & Q & A & K & G & I & Q & L\end{array}$ 481 ATGGAAGAGAATGAACGGTTAAGACAGCAAGTGGTGGAGATCACTAGTGGCCGGAGACGT

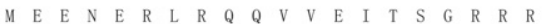
541 GTTGGTGGTGATTCGGAAAATGTGGTTAACGAGGAAGGCCAGTCATCAGAGTCGGTTACC $\begin{array}{lllllllllllllllllllll}V & G & G & D & S & E & N & V & V & N & E & E & G & Q & S & S & E & S & V & T\end{array}$

601 AATCATTGCAATTCCACTGGTCCCCCACATGACTATGAAAGCTCAGATACATCTCTCAAG

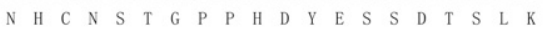
661 TTAGGCTTACCCTATGGTGGTTAA

L $\quad$ G

LcSVP2 (1-642)

1 ATGACGAGACAGAAAATTGAGATCAaGAagATCGACAACACCACGGCCAGGCAGGTTACA

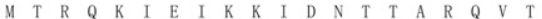
61 TTCTCAAAAAGGAGAaGAGGGCTTTTCAAGAAGGCACAGGAGCTCTCAACTCTCTGTGAT $\begin{array}{lllllllllllllllllllll}F & S & K & R & R & R & G & L & F & K & K & A & Q & E & L & S & T & L & C & D\end{array}$

121 GCTGAAATAGCTCTCATGGTCTTCTCTGCCACAGGAAAGCTCTTTGAATACTCCAGCTCA

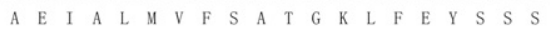
181 AgtaCGaAACAGGTGATTGAAAAATACAAGATGAaTATGCCGAAACTTCACAGATTCGAT S T K K Q 241 CAACCTTCTCTAGAATTACAGCTTGAATGCAATACCTATGCCATGTTGAGCAGAGAAATT

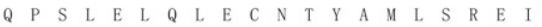

301 GCCGAAAAGACCCGTGAGCTAAGGCAGATGAGGGGAGAAGAACTCCAAGGGTTGAATGTA

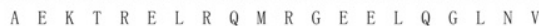

361 GAGGAGCTAAAGGAACTAGAAAAATCACTTGAAGGAGGGTTAAGATGTGTGGCCGAAACG

421 AagGgtGaAagaATTATGAaAgAaATTAGTGAGCTCAAGACAAGGGGACAGCAGCTGATG K G 481 GATGAAAATCTGAGACTGAGGCAAAGAACACGGAATTTAGATTCACATGTTCAAGGCCAG $\begin{array}{lllllllllllllllllllll}\text { D } & E & N & L & R & L & R & Q & R & T & R & N & L & D & S & H & V & Q & G & Q\end{array}$ 541 TCGTCAGAGTCAGTTACCAATATCAGCATCTTAGGAGATGTTCCTCAAGACAGTAACAGC

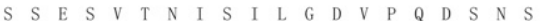
601 TCTGATACTTCTTTAAAGTTGGGGTTGCATTTCCTAGCTAA

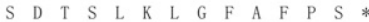

$1 \mathrm{csvp} 3(1-681)$

1 ATGGCGAGAGAGAaGATCAAGATCAAGAaGATTGACAACGTGACGGCCAGGCAAGTCACC

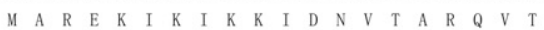
61 TTCTCCAAGCGAAGACGTGGACTTTTCAAGAAAGCCGAAGAGCTTTCTGTTCTCTGCGAC

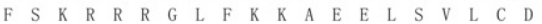
121 GCTGAAGTTGCTCTCATCATCTTCTCAGCTACTGGCAAGCTCTTTGAGTATTCAAGCTCC

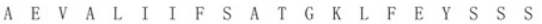

181 AGCATGAAGGATATAATTTCACGGTATAATCTGCACTCAAGTAACCTGGGCAAATTGAAC

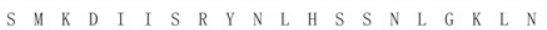
241 CAACCTTCTCTTGAACTGCAGCTGGAAAATAGCAACCACATGAGATTGAGTAAGGAAATT Q $\begin{array}{llllllllllllllllllll} & \text { S } & L & E & L & Q & L & E & N & S & N & H & M & R & L & S & K & E & I\end{array}$ 301 GCTGATAAGAGCCAACAACTGAGGCAGATGAGAGGAGAGGATCTTCAAGGATTGAAAATA

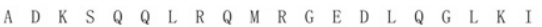

361 GAGGAATTACAACAACTTGAGAAAATGCTTGAATCAGGACTTAGCCGTGTGATTGAGACT

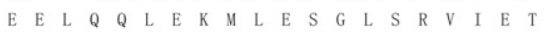
421 AAGGGTGAACGGATTATTAATGAGATTACAACACTCGAAAGAAAGGGAGTTCAGCTGTTA K $\quad$ G 481 GAAGAGAACAAGCAACTAAAACAAAGGGTGGACGTGTTAAAGGGAAAAAGAGTCGTCTTC

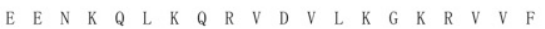

541 ATGGAGTCGGATATTGGTATCCAGGAAGAAGGGTTGTCTTCGGAATCTGCTACCAACGTA $\begin{array}{llllllllllllllllllll}M & E & S & D & I & G & I & Q & E & E & G & L & S & S & E & S & A & T & N & V\end{array}$ 601 TGCAGCTGCAGCAGTGGCCCTCCTCTAGAGGATGATAGTTCTGATACTTCTCTTAAATTA

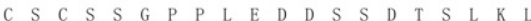
661 GGGCTTCCCTTCTCTAGTTGA

G $\quad$ L

Supplemental Fig. 1. Deduced amino acid sequence of the short vegetative phase (SVP) homologs $(L c S V P \mathrm{~s}) \mathrm{cDNA}$ in litchi.

J. Amer. Soc. Hort. Sci. 143(2):1-3. 2018. 


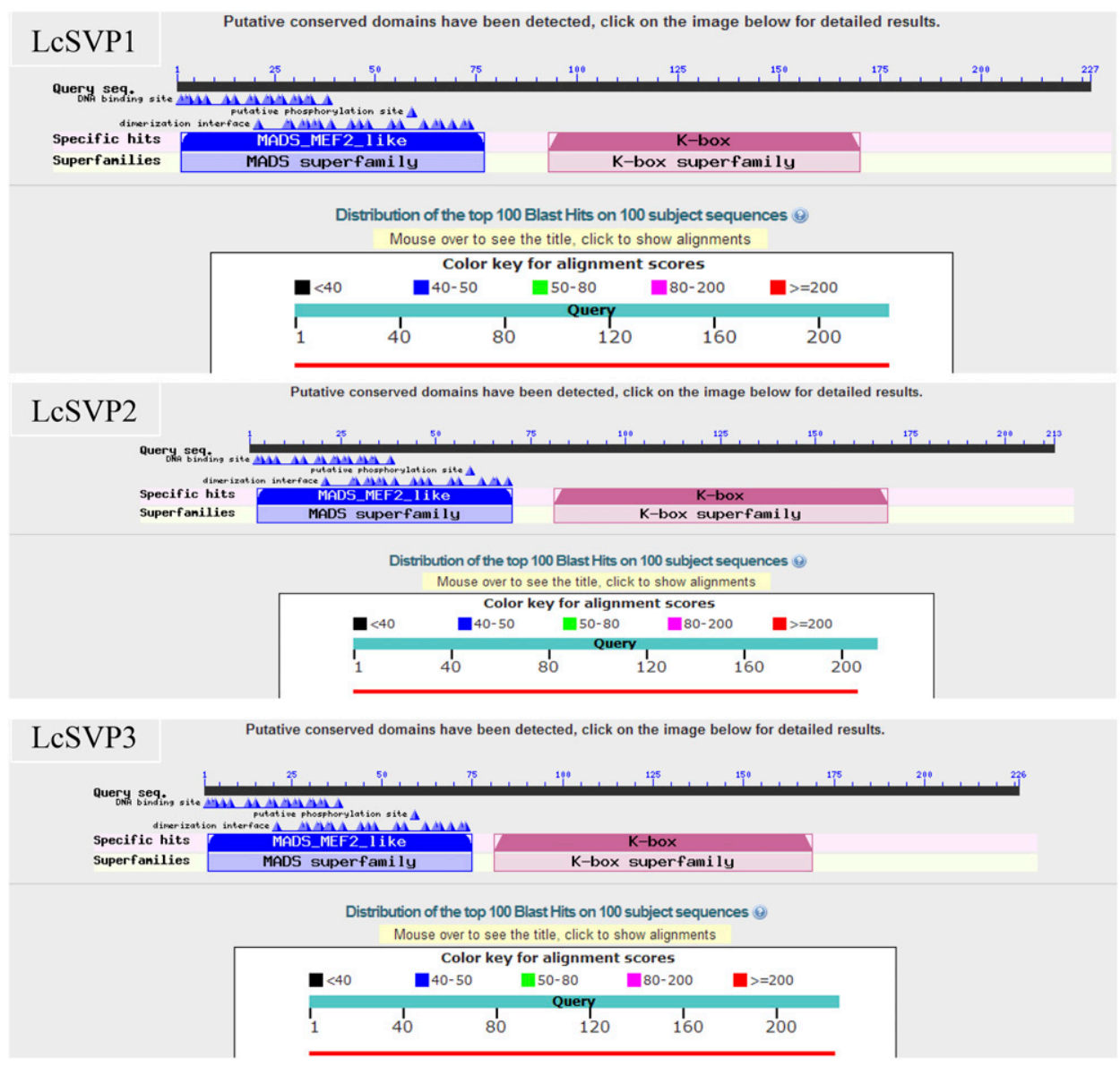

Supplemental Fig. 2. NCBI BLASTp results of the short vegetative phase (SVP) homologs LcSVP1, LcSVP2, and LcSVP3 in litchi. 
Predicted Secondary Structure (3 Class): LcSVP1

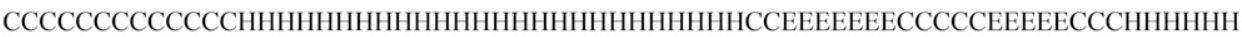

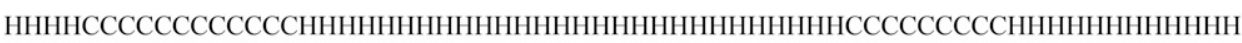

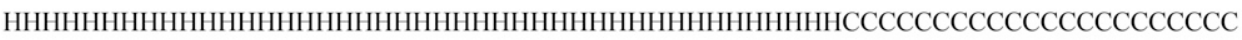

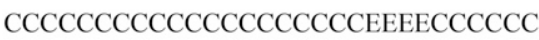

Predicted Secondary Structure (3 Class):LcSVP2

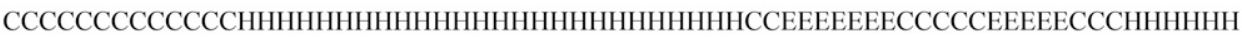

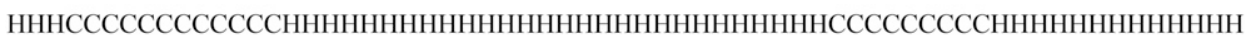

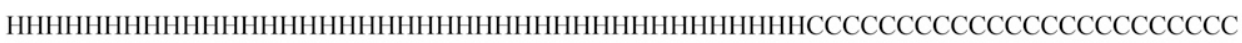
CCCCCCEEEECCCCCC

Predicted Secondary Structure (3 Class):LcSVP3

СССCCCCCCCCCHHHHHHHHHHHHHHHHHHHHHHHHHHCCEEEEEEECCCCCEEEEECCCHHHHHH

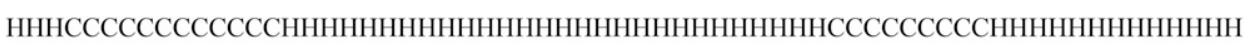

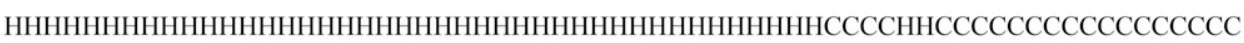
CCCCCCCCCCCCCCCCCCCEEEECCCCCC

Supplemental Fig. 3. Prediction of the secondary structure of the short vegetative phase $(S V P)$ homologs $L c S V P 1, L c S V P 2$, and $L c S V P 3$ in litchi. $\mathrm{H}=$ alpha helice; $\mathrm{E}=$ beta turn; $\mathrm{C}=$ random coil.
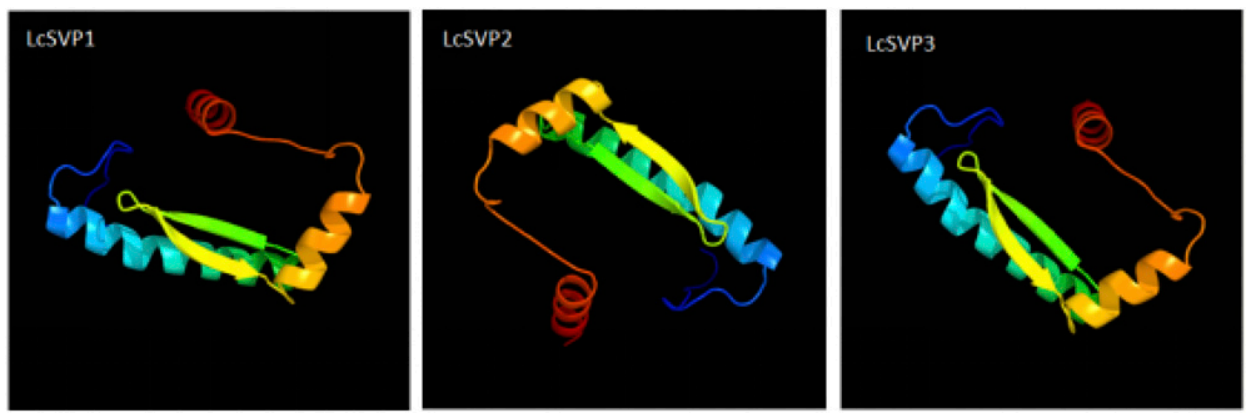

Supplemental Fig. 4. Tertiary structure prediction of the short vegetative phase (SVP) homologs $L c S V P 1, L c S V P 2$, and $L c S V P 3$ in litchi. 